\title{
Evaluating Elimination Thresholds and Stopping Criteria for Interventions Against the Vector-borne Macroparasitic Disease, Lymphatic Filariasis, Using Mathematical Modelling
}

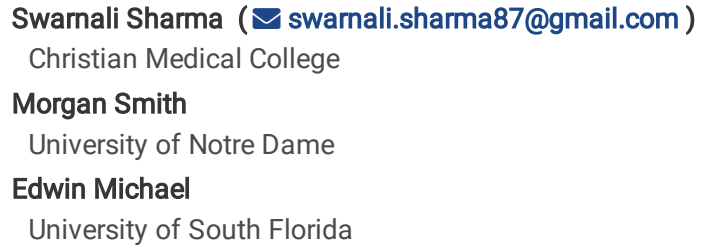

Article

Keywords: EPIFIL, TAS strategy, Transmission Assessment Surveys (TAS)

Posted Date: September 30th, 2021

DOI: https://doi.org/10.21203/rs.3.rs-898556/v1

License: @ (i) This work is licensed under a Creative Commons Attribution 4.0 International License. Read Full License

Version of Record: A version of this preprint was published at Communications Biology on February 27th, 2023. See the published version at https://doi.org/10.1038/s42003-022-04391-9. 


\section{Abstract}

We leverage the ability of the EPIFIL transmission model fit to field data for allowing calculations of the probabilities of transmission elimination and recrudescence once infection levels are predicted to fall below the threshold used in the WHO Transmission Assessment Surveys (TAS) versus site-specific model-estimated thresholds to evaluate the implications of using these thresholds for making Lymphatic Filariasis (LF) intervention stopping decisions. Our results, overall, indicate that understanding the underlying parasite transmission and extinction dynamics will be crucial for choosing the right intervention stopping thresholds, and indeed the right interventions connected with these thresholds, if we are to bring about the sustainable elimination of LF. They also warn that applying stopping criteria set for operational purposes without a full consideration of population dynamics, as employed in the current TAS strategy, could, by risking infection recrudescence especially over the long-term, seriously undermine the goal of achieving global LF elimination.

\section{Introduction}

Lymphatic filariasis (LF), a highly debilitating mosquito-borne macroparasitic disease of humans, is only one of eight neglected tropical diseases (NTDs) selected by the World Health Organization (WHO) for either elimination or eradication as a global public health problem ${ }^{1}$. This resulted in the development of the Global Program to Eliminate Lymphatic Filariasis (GPELF), which espoused meeting the goal of eliminating this disease in all 73 endemic countries by 2020 through the application of annual mass drug administration (MDA) maintained over at least 4-6 years ${ }^{1}$. GPELF is not only one of the largest global intervention programs devised to reduce the burden of tropical diseases, but since its origin the program has also made remarkably effective strides in reducing infection prevalence in all the endemic countries in which large-scale nation-wide LF MDA programs have been implemented ${ }^{2}$. This striking success means that many LF programs are now beginning to enter into the endgame or pre-elimination phase of intervention, with some also considering scaling down MDA to move into a period of post-MDA surveillance that will culminate with a request to WHO to verify the achievement of country-wide elimination of the disease ${ }^{3}$.

While the above progress has highlighted the effectiveness of GPELF for successfully reducing the burden of LF in endemic populations, it has also raised increasing attention on how best to define and implement robust strategies for supporting decisions to end interventions. In 2011 , the WHO recommended the use of an epidemiological assessment strategy that comprises conducting a series of infection surveys of entire communities initially, and subsequently of children aged 6-7 years in treated communities, to provide evidence for deciding if transmission has been interrupted and so enable the making of defensible intervention stopping decisions ${ }^{4}$. The first survey in this strategy, termed a pre-Transmission Assessment Survey (TAS) or pre-TAS, is to be undertaken in all sentinel and spot check sites of implementation units (IUs) - normally these are districts - which have had at least five effective (>65\% coverage) rounds of MDA to evaluate if community-level prevalences of either microfilaraemia (mf) or circulating antigen (CFA) have reached below $1 \%$ or $2 \%$ respectively. If either of these targets is met in all the surveyed communities, then an IU is deemed eligible for initiating TAS surveys of children aged 6-7 years sampled from across populations in an IU ${ }^{5}$. In this scheme, the first TAS is designed to be conducted at least six months after the final round of MDA to decide if drug treatments can be stopped, while subsequent TAS surveys are conducted (at 2-3 year intervals) to establish the absence of ongoing transmission. Transmission is considered not sustainable when over the period of TAS survey bouts (ie. at least over a period of 5 years after stopping MDA) the mean antigen (CFA) prevalence in the sampled children drops and remains essentially below half the community-wide thresholds mentioned above, ie. $1 \%$ (or equivalently $<0.5 \% \mathrm{mf}$ ) in areas where Anopheles, Culex and Mansonia species are the main mosquito vectors ${ }^{4}$.

The above strategy has been integrated into national LF programs since 2011, and is widely thought among managers to represent a standardized, statistically rigorous, and easily implementable tool for guiding LF intervention stopping decisions; however, increasing evidence from the field ${ }^{6-8}$ and results from recent modelling work ${ }^{9-12}$ have called to question whether meeting the stopping criteria used by $1 \%$ mf threshold recommended by WHO or TAS would indeed signify the breakage of LF transmission in endemic communities and hence if MDA can be safely terminated following this strategy. Specifically, while the available field studies that have evaluated the impact of TAS show that passing TAS may not allow evaluation of ongoing residual transmission nor provide a good indication of prevalence levels in older age groups ${ }^{7,13}$, the globally applicable thresholds used in this protocol do not also reflect the likely much lower transmission interruption values and the site-to-site variation estimated for these LF breakpoints by data-driven mathematical models 9,10 . Furthermore, the TAS strategy appears to be devised such that the threshold values chosen are sufficiently low to presume that breaching them would lead to transmission interruption, whilst also serving simultaneously to allow the setting and use of sample sizes that are thought to be achievable in the field ${ }^{14}$.

The above suggests that the WHO recommended 1\% elimination threshold or the TAS protocol may be deemed to serve as a practical framework good enough for meeting operational purposes; however, it is clear given the growing discrepant evidence that it is also important to determine whether it provides the right results for the right reasons, viz. that meeting the currently set TAS criteria will indeed lead to cessation of parasite transmission. This concern at its core questions whether crossing the present WHO-set TAS thresholds actually denote the achievement of sustained interruption of LF transmission (defined as infection found to be consistently below $0.5 \% \mathrm{mf}$ or $1 \%$ CFA in children during the TAS phase ${ }^{3}$ ). If they do not, then as mathematical models and growing evidence from the field show ${ }^{6-12}$, recrudescence of infection can be expected which will seriously thwart the goal of successfully achieving the global elimination of this parasitic disease.

Parasite transmission models aim to encapsulate our understanding of the non-linear, non-additive behaviour of a parasitic system, and thus provide powerful tools for quantifying the dynamical impact of interventions, including affording predictions of when infection is reduced below thresholds that signify that transmission in nature has been broken ${ }^{3,9,10}$. As these models aim to reproduce system dynamics based on our understanding of how a given parasitic system operates, they can also serve as a major tool for determining if we are getting the right results for the right reasons when addressing a policy objective. Additionally, developing and applying data-driven prediction frameworks that allow coupling of information on local system dynamics as provided by field observations with models means that that we are able to more consistently get scientifically consistent answers for meeting policy objectives equally well everywhere 9,10 
In this study, we leverage the ability of mathematical models fit to field data for allowing direct calculations of probabilities of transmission elimination once infection levels are forecast to fall below given thresholds to evaluate: 1) the implications of using the WHO TAS criteria for assessing the achievement of LF transmission interruption, and conversely, 2) the likely probability of transmission recrudescence if elimination of transmission is not achieved. The deterministic Monte Carlo population-based LF model, EPIFIL ${ }^{9,10}$, calibrated to longitudinal human infection data from six field studies that encompass endemic settings typifying the full range of low, moderate and high LF transmission intensities and which also provided MDA intervention details, was used to carry out the simulations reported in this work. Predictions of the impacts of MDA-based interventions are compared between using the WHO-set global TAS versus model-estimated thresholds in these sites in order to highlight the significance of using breakpoint values more explicitly linked to the underlying localized parasite transmission dynamics in a setting compared to those more generally or globally set. Finally, we also examine the relative effectiveness of currently existing or proposed MDA-based strategies for best ensuring the achievement of LF transmission interruption while also suppressing the probability of recrudescence, paying particular attention to the role that supplementary vector control can play for meeting both these outcomes.

\section{Results}

\section{Estimating local LF models and assessment of predictive performance}

We began by assessing the ability of our BM-based data-model fusion modelling approach for successfully estimating best-fitting LF models in each of the present study sites. Note that age-stratified $\mathrm{mf}$ prevalences had to be constructed for 4 of these sites that provided only overall community mf prevalence observations ${ }^{15,16}$, and all $\mathrm{mf}$ prevalence values were corrected to reflect sampling of $1 \mathrm{ml}$ blood volumes for carrying out this exercise ${ }^{15,17}$. Nonetheless, the curves in Fig. 1 show that the LF models identified for each site based on the SIR algorithm are indeed well able to satisfactorily reproduce the site-varying baseline mf infection prevalence observed for either of these types of data - ie. whether actually measured (circles) or constructed (circles joined by lines) (Monte Carlo- $p$-values $>0.05$ for each site except in the case of Piapung and Kirare (Table S2 in Supplementary Information)).

The predictive performances of these locally data-informed models were assessed by comparing forecasts of the impacts of the specific MDAs carried out in each site (Table 1) for reducing mf prevalence against the corresponding actually observed reductions in these data per site (Fig. 2). These comparisons indicate that, as with predictions of site-specific baseline infection prevalences (Fig. 1), and apart from a tendency to overestimate prevalence reductions at some time points in the Piapung and Kirare sites, the estimated locally applicable LF models are also able to mimic the observed changes in the data due to the applied interventions sufficiently well (Monte Carlo $p$-values for assessing goodness-of-fit insignificant in each setting $>0.05$ (Table $\mathrm{S} 2$ in Supplementary Information)).

\section{Estimating timelines and probabilities of elimination due to the MDA alone interventions carried out in each site}

The numerical forecasts of the timelines made by each site-specific model ensemble for reaching the $1 \%$ mf prevalence threshold due to the annual MDAs applied (at the average coverages reported in Table 4) in each of the present study sites are given in Table 1A. These highlight that apart for the case of Piapung the present models are able to reliably predict the required MDA intervention durations for breaching the $1 \%$ mf target in each site. Thus, where the number of years predicted by the locality-specific models for achieving the $1 \% \mathrm{mf}$ threshold were either lower or equal to the actually applied annual MDA rounds, the final prevalences observed (following the applied MDAs) in these sites were found to be lower than the $1 \% \mathrm{mf}$ prevalence threshold (Table $1 \mathrm{~A}$ ). This is in contrast to sites (eg. Kirare, Peneng) in which the model predicted annual MDA durations for reaching the $1 \%$ mf threshold are estimated to be longer than the actually applied MDA durations; in this case the end $\mathrm{mf}$ prevalences reached are, as expected, found to be higher than the $1 \% \mathrm{mf}$ prevalence target (Table 1). This matching of predictions with observations futher confirm the conclusion made above that the present locally calibrated models are empirically adequate for capturing the dynamics of the applied MDA control in each site, and so can serve as reliable tools for addressing the chief objective of this study, viz. evaluating the consequences of applying the WHO TAS criteria for guiding decision making regarding the site-specific interruption of LF transmission.

Table 1 also provides the elimination probabilities over the 5-year TAS period estimated by the localized predictive models following the reduction of mf prevalences below the $1 \% \mathrm{mf}$ threshold (Table $1 \mathrm{~A}$ ) or the significantly much lower model-estimated $95 \%$ EP mf prevalence thresholds estimated at ABR in each of the present sites (Table 1B). The calculated probabilities show that while achieving the $1 \% \mathrm{mf}$ prevalence target in each site will require significantly fewer years of annual MDAs (from 3 to 9 years depending on intial endemicity), such achievement will, however, result in either low or moderate chance (4 to $50 \%$ ) of transmission interruption with a corresponding significant probabilities of recrudescence (between 50 to $96 \%$ ) (Table $1 \mathrm{~A}$ ). By contrast, even though it will take considerably longer (11 to 19 years) to achieve the much lower $95 \%$ EP thresholds (ranging from $0.001-0.007 \%$ ) applicable in each site, the results show that once these thresholds are crossed, the probability of achieving elimination of transmission will be very high and close to nearly $100 \%$ ( $>89 \%$ ) in each site, with the corresponding recrudescence probabilities effectively close to zero, except in the case of Piapung village (Table 1B). 
Table 1

A: Observed and model predicted post-MDA data for WHO recommended 1\% mf threshold for six LF infected sites.

\begin{tabular}{|c|c|c|c|c|c|c|}
\hline Settings & Site & $\begin{array}{l}\text { No. of annual } \\
\text { MDA rounds } \\
\text { observed }\end{array}$ & $\begin{array}{l}\text { Mf prevalence } \\
\text { following } \\
\text { interventions }\end{array}$ & $\begin{array}{l}\text { Mean No. of years to } \\
\text { reach } 1 \% \mathrm{mf} \text { (model } \\
\text { predicted) }\end{array}$ & $\begin{array}{l}\text { Probability of elimination (\%) } \\
\text { 5years after crossing } 1 \% \mathrm{mf} \\
\text { threshold }\end{array}$ & $\begin{array}{l}\text { Probability of recrudescence } \\
\text { (\%) 5years after crossing } 1 \% \\
\text { mf threshold }\end{array}$ \\
\hline \multirow[t]{2}{*}{ Low } & $\begin{array}{l}\text { DokanTofa, } \\
\text { Nigeria }^{18}\end{array}$ & 7 & $0.4 \%$ & 3 & 9 & 91 \\
\hline & $\begin{array}{l}\text { Piapung, } \\
\text { Nigeria }^{18}\end{array}$ & 7 & $2.1 \%$ & 4 & 4 & 96 \\
\hline \multirow[t]{2}{*}{ Medium } & $\begin{array}{l}\text { Missasso, } \\
\text { Mali19 }^{19}\end{array}$ & 6 & $0.0 \%$ & 5 & 8 & 92 \\
\hline & $\begin{array}{l}\text { Kirare, } \\
\text { Tanzania } 20\end{array}$ & 6 & $2.7 \%$ & 7 & 20 & 80 \\
\hline \multirow[t]{2}{*}{ High } & $\begin{array}{l}\text { Peneng, } \\
\text { PNG }^{21}\end{array}$ & 5 & $3.7 \%$ & 9 & 50 & 50 \\
\hline & $\begin{array}{l}\text { Dozanso, } \\
\text { Mali }^{19}\end{array}$ & 6 & $0.0 \%$ & 6 & 32 & 68 \\
\hline
\end{tabular}

Table 1

B: Model predicted post-MDA data for model predicted 95\% EP threshold for six LF infected sites.

\begin{tabular}{|lllllll|}
\hline Settings & Site & $\begin{array}{l}\text { 95\% EP } \\
\text { threshold } \\
\text { values }\end{array}$ & $\begin{array}{l}\text { Mean no. of years to reach } \\
\text { 95\% EP threshold (model } \\
\text { predicted) }\end{array}$ & $\begin{array}{l}\text { Probability of elimination (\%) 5years } \\
\text { after crossing model predicted 95\% EP } \\
\text { threshold }\end{array}$ & $\begin{array}{l}\text { Probability of recrudescence (\%) 5years } \\
\text { after crossing model predicted 95\% EP } \\
\text { threshold }\end{array}$ \\
\hline Low & $\begin{array}{l}\text { DokanTofa, } \\
\text { Nigeria }\end{array}$ & 0.003109 & 11 & 95 & 5 \\
\hline & $\begin{array}{l}\text { Piapung, } \\
\text { Nigeria }\end{array}$ & 0.001550 & 13 & 89 & 11 \\
\hline Medium & $\begin{array}{l}\text { Missasso, } \\
\text { Mali }\end{array}$ & 0.007500 & 15 & 98 & 2 \\
\hline $\begin{array}{l}\text { Kirare, } \\
\text { Tanzania }\end{array}$ & 0.001799 & 18 & 98 & 2 \\
\hline High & $\begin{array}{l}\text { Peneng, } \\
\text { PNG }\end{array}$ & 0.004534 & 19 & 99 & 1 \\
\hline $\begin{array}{l}\text { Dozanso, } \\
\text { Mali }\end{array}$ & 0.001101 & 16 & 95 & 5 \\
\hline
\end{tabular}

Assessments of the impact of including vector control in MDA interventions on elimination and recrudescence probabilities

We next examined the relative effectiveness of three MDA scenarios with and without inclusion of VC for accomplishing sustainable LF elimination and reducing likely recrudescence in the present sites. The three MDA-based scenarios chosen for study were: (i) annual MDA, (ii) biannual MDA and (iii) annual IDA, firstly, with and without inclusion of supplementary VC from the beginning of MDA to when the WHO proposed $1 \%$ mf prevalence or model-estimated $95 \%$ EP thresholds are breached, and, secondly, after achievement of these thresholds using MDA continuing with VC alone for either over the 5-year TAS period or for up to 20 years post stoppage of MDA (Table 2). The drug combinations applied in each site (ie. IVM + ALB or IVM + DEC (Table 4)) were used for simulating the impacts of annual and biannual MDA respectively in each site, while the combined effects of IVM, DEC and ALB were modelled for forecasting the impact of annual IDA. The average MDA coverage and baseline mf prevalence pertaining to each site were used to run these scenario simulations (see Methods).

The results from carrying out the various MDA only and MDA plus VC intervention simulations are shown in the case of Piapung, Missaso and Dozanso villages, representing the low, medium and high baseline endemic sites investigated here respectively, for both the $1 \%$ (Table $2 \mathrm{~A}$ ) and their corresponding sitespecific 95\% EP mf prevalence thresholds (Table 2B). The time in years to cross each respective threshold and the probabilities of achieving elimination or recrudescence following reaching either threshold (and stoppage of MDA) are depicted in these tables with and without inclusion of VC for both the 5-year TAS period as well as over a longer 20-year term following the breaching of these thresholds. The results demonstrate, firstly, as expected, that timelines to reach the $1 \% \mathrm{mf}$ theshold will increase with endemicity but decreased in each of the three sites as annual treatments are replaced by biannual two-drug MDAs and by the annual administration of IDA irrespective of whether VC is included or not (Table 2A). Thus, while annual single drug MDA interventions (with or without $\mathrm{VC}$ ) took the longest times to reach the $1 \% \mathrm{mf}$ prevalence target among the present drug interventions, this threshold was reached earlier in the the low transmission village of Piapung (5 years) compared to the need for 9 years of annual mass treatments required for Dozanso with the same IVM + ALB regimen. Biannual MDA, however, is predicted to be the most effective strategy for achieving the $1 \% \mathrm{mf}$ threshold in the present villages, given that it takes only 1 (Piapung) to 4 years (Dozanso) compared to the 2 to 5 years required by annual IDA to cross this threshold in the same villages (Table $2 A$ ).

The second finding arising from the results depicted in Table $2 \mathrm{~A}$ is that using annual MDAs, irrespective of the drug regimens used and inclusion of $\mathrm{VC}$, counterintuitively resulted in greater elimination (between 4-51\% probability of elimination after 5 years and between 26 to 100 probability of elimination after 20 years) and lower recruscrence (between $49-96 \%$ recrudescence probabilities after 5 years; between $0-74 \%$ recrudescence probabilities after 20 years) in 
these 3 villages compared to the application of the biannual MDA and IDA interventions (elimination probabilities generally between 0-33\% with corresponding recrudescence probabilities reaching generally $>70-100 \%$ ) following the achievement of the $1 \%$ mf threshold (Table $2 \mathrm{~A}$ ).

Combining VC with MDA from the start until the $1 \% \mathrm{mf}$ threshold is reached did not overly improve the results obtained with the use of MDA alone, irrespective of the MDA strategy; however, including VC over the long-term (ie for 20 years after achievement of the $1 \%$ mf threshold by MDA) had an dramatic effect in increasing the probability of elimination (to $>71 \%$ ) and reducing the corresponding probability of recrudescence (to as low as $<10 \%$ ) depending on site and MDA strategy. Including VC after achievement of the $1 \% \mathrm{mf}$ threshold by MDA for over the 5 -year TAS period alone, however, while improving the results obtained with the use of MDA alone irrespective of site or MDA strategy (Table 2A), resulted in lower elimination probabilities compared to when it was included over the longer 20-year period for all interventions. As a result of this, all estimated recruscence probabilities were found to be higher for the 5-year TAS period compared to the corresponding values calculated for the longer term 20-year period, highlighting the need for longer continuation with VC beyond the 5-year TAS period to ensure the breakage of parasite transmission under this scenario.

The corresponding results on timelines to extinction and probabilities of elimination and recrudescence using the corresponding site-specific $95 \%$ EP thresholds as the extinction target in each village is shown in Table 2B. These show that using these thresholds will first of all significantly lengthen the durations of interventions required to break parasite transmission. Thus, while using annual single MDAs will take between 14 to 24 years to cross these thresholds in the three example villages - as compared to just 5 to 9 years it would take for this intervention to cross the $1 \%$ mf threshold in the same villages depending on site and inclusion of VC (Table 2A) - this will be reduced to between 8 to 13 and 10 to 17 years of treatments required when the corresponding biannual and annual IDA MDA strategies are used in these villages (in comparison with just the 1 to 4 and 2 to 5 years required by these interventions to reach the $1 \% \mathrm{mf}$ threshold ). Adding VC to each MDA strategy from the beginning, by contrast to the results arising from using the $1 \% \mathrm{mf}$ threshold, however, despite still taking longer, will result in reducing the overall durations required (by at least 2 to 6 years depending on strategy and site) to reach the $95 \%$ EP threshold (Table 2B).

The estimated probabilities of elimination and recrudescence depicted in Table 2B, on the other hand, highlight the major benefit of using the 95\% EP threshold as targets for LF elimination programs, viz. that once these thresholds are breached by an intervention, it will inevitably lead to interruption of transmission (>98\% probability at least) and result in the occurrence of close to zero probabilities of infection recrudescence, particularly over the longer 20year period irrespective of site or type of MDA-based strategy (Table 2B). Another interesting, but perhaps expected finding, is that simply using MDA alone for achieving the $95 \%$ EP threshold, in contrast to using the $1 \% \mathrm{mf}$ target, is also adequate for accomplishing transmission elimination as well as reducing the probability of infection recrudescence to close to zero over the long-term irrespective of the vagaries of site conditions and whether VC is included or not (compare Tables 2B and A). However, increasing the frequency of MDA (to biannual treatments) and use of annual IDA resulted in lower elimination and higher recrudescence probabilities following the 5-year TAS period irrespective of whether VC was included or not when these $95 \%$ EP targets are used (Table 2B)

Inclusion of VC with MDA from the beginning helped to reduce the time to reach the site-specific $95 \%$ EP thresholds and to increase the probability for transmission elimination for the 5-year TAS period, but it was found not to add any additional benefit to increasing the probability for achieving transmission elimination or reducing the probability of recrudescence over that accomplished by MDA alone (carried out until the $95 \%$ EP thresholds are reached) for a longer term 20 years period (Table 2B), in contrast to these outcomes when using the $1 \% \mathrm{mf}$ threshold (Table 2A). Applying VC during 5 years (TAS period) after achievement of the $95 \%$ EP threshold by MDA alone will also result in almost the same benefit in increasing the probability for achieving transmission elimination or reducing the probability of recrudescence as that accomplished by carrying out either MDA alone or by implementing longer-term supplementary VC (Table 2B). 
Table 2

A. Recrudescence probability and elimination probability for different scenarios with $1 \% \mathrm{mf}$ threshold for one low (Piapung), one med (Missasso) and one high prev (Dozanso) sites.

\begin{tabular}{|c|c|c|c|c|c|c|c|c|c|c|c|c|c|c|c|}
\hline \multirow[b]{3}{*}{ Scenario } & \multicolumn{5}{|l|}{ Piapung } & \multicolumn{5}{|l|}{ Missaso } & \multicolumn{5}{|l|}{ Dozanso } \\
\hline & \multirow[b]{2}{*}{$\begin{array}{l}\text { Time to } \\
\text { cross } \\
\text { threshold } \\
\text { (year) }\end{array}$} & \multicolumn{2}{|c|}{$\begin{array}{l}\text { Elimination } \\
\text { probability } \\
\text { (\%) }\end{array}$} & \multicolumn{2}{|c|}{$\begin{array}{l}\text { Recrudescence } \\
\text { probability (\%) }\end{array}$} & \multirow[b]{2}{*}{$\begin{array}{l}\text { Time to } \\
\text { cross } \\
\text { threshold } \\
\text { (year) }\end{array}$} & \multicolumn{2}{|c|}{$\begin{array}{l}\text { Elimination } \\
\text { probability } \\
(\%)\end{array}$} & \multicolumn{2}{|c|}{$\begin{array}{l}\text { Recrudescence } \\
\text { probability (\%) }\end{array}$} & \multirow[b]{2}{*}{$\begin{array}{l}\text { Time to } \\
\text { cross } \\
\text { threshold } \\
\text { (year) }\end{array}$} & \multicolumn{2}{|c|}{$\begin{array}{l}\text { Elimination } \\
\text { probability } \\
\text { (\%) }\end{array}$} & \multicolumn{2}{|c|}{$\begin{array}{l}\text { Recrudescence } \\
\text { probability (\%) }\end{array}$} \\
\hline & & $\begin{array}{l}\text { After } \\
5 \\
\text { years }\end{array}$ & $\begin{array}{l}\text { After } \\
35 \\
\text { years }\end{array}$ & $\begin{array}{l}\text { After } \\
5 \\
\text { years }\end{array}$ & $\begin{array}{l}\text { After } \\
35 \\
\text { years }\end{array}$ & & $\begin{array}{l}\text { After } \\
5 \\
\text { years }\end{array}$ & $\begin{array}{l}\text { After } \\
35 \\
\text { years }\end{array}$ & $\begin{array}{l}\text { After } \\
5 \\
\text { years }\end{array}$ & $\begin{array}{l}\text { After } \\
35 \\
\text { years }\end{array}$ & & $\begin{array}{l}\text { After } \\
5 \\
\text { years }\end{array}$ & $\begin{array}{l}\text { After } \\
35 \\
\text { years }\end{array}$ & $\begin{array}{l}\text { After } \\
5 \\
\text { years }\end{array}$ & $\begin{array}{l}\text { After } \\
35 \\
\text { years }\end{array}$ \\
\hline $\begin{array}{l}\text { Annual } \\
\text { MDA } \\
\text { upto } 1 \% \\
\text { mf }\end{array}$ & 5 & 4 & 33 & 96 & 67 & 6 & 8 & 26 & 92 & 74 & 9 & 32 & 48 & 68 & 52 \\
\hline $\begin{array}{l}\text { Annual } \\
\text { MDA + } \\
\text { VC upto } \\
1 \% \text { mf }\end{array}$ & 5 & 5 & 36 & 95 & 64 & 6 & 13 & 33 & 87 & 67 & 9 & 39 & 54 & 61 & 46 \\
\hline $\begin{array}{l}\text { Annual } \\
\text { MDA } \\
\text { upto 1\% } \\
\text { mf, } \\
\text { endgame } \\
\text { VC }\end{array}$ & 5 & 23 & 99 & 77 & 1 & 6 & 40 & 100 & 60 & 0 & 9 & 51 & 99 & 49 & 1 \\
\hline $\begin{array}{l}\text { Annual } \\
\text { MDA } \\
\text { upto } 1 \% \\
\text { mf, then } \\
\text { only VC } \\
\text { for next } \\
\text { 5years }\end{array}$ & 5 & 23 & 58 & 77 & 42 & 6 & 40 & 48 & 60 & 52 & 9 & 51 & 61 & 49 & 39 \\
\hline $\begin{array}{l}\text { Biannual } \\
\text { MDA } \\
\text { upto 1\% } \\
\text { mf }\end{array}$ & 1 & 0 & 0 & 100 & 100 & 3 & 2 & 14 & 98 & 86 & 4 & 6 & 21 & 94 & 79 \\
\hline $\begin{array}{l}\text { Biannual } \\
\text { MDA + } \\
\text { VC upto } \\
1 \% \text { mf }\end{array}$ & 1 & 0 & 0 & 100 & 100 & 3 & 2 & 16 & 98 & 84 & 4 & 7 & 23 & 93 & 77 \\
\hline $\begin{array}{l}\text { Biannual } \\
\text { MDA } \\
\text { upto 1\% } \\
\text { mf, } \\
\text { endgame } \\
\text { VC }\end{array}$ & 1 & 0 & 71 & 100 & 29 & 3 & 12 & 89 & 88 & 11 & 4 & 10 & 82 & 90 & 18 \\
\hline $\begin{array}{l}\text { Biannual } \\
\text { MDA } \\
\text { upto } 1 \% \\
\text { mf, then } \\
\text { only VC } \\
\text { for next } \\
\text { 5years }\end{array}$ & 1 & 0 & 3 & 100 & 97 & 3 & 12 & 30 & 88 & 70 & 4 & 10 & 33 & 90 & 67 \\
\hline $\begin{array}{l}\text { Annual } \\
\text { IDA upto } \\
1 \% \mathrm{mf}\end{array}$ & 2 & 0 & 0 & 100 & 100 & 4 & 0 & 5 & 100 & 95 & 5 & 0 & 4 & 100 & 96 \\
\hline $\begin{array}{l}\text { Annual } \\
\text { IDA + VC } \\
\text { upto } 1 \% \\
\text { mf }\end{array}$ & 2 & 0 & 0 & 100 & 100 & 4 & 0 & 6 & 100 & 94 & 5 & 0 & 11 & 100 & 89 \\
\hline $\begin{array}{l}\text { Annual } \\
\text { IDA upto } \\
1 \% \mathrm{mf}, \\
\text { endgame } \\
\text { VC }\end{array}$ & 2 & 0 & 76 & 100 & 24 & 4 & 0 & 92 & 100 & 8 & 5 & 0 & 80 & 100 & 20 \\
\hline $\begin{array}{l}\text { Annual } \\
\text { IDA upto } \\
1 \% \text { mf, } \\
\text { then only } \\
\text { VC for } \\
\text { next } \\
\text { 5years }\end{array}$ & 2 & 0 & 6 & 100 & 94 & 4 & 0 & 15 & 100 & 85 & 5 & 0 & 15 & 100 & 85 \\
\hline
\end{tabular}


Table 2

B. Recrudescence probability and elimination probability for different scenarios with 95\% EP threshold for one low (Piapung), one med (Missasso) and one hig prev (Dozanso) site

\begin{tabular}{|c|c|c|c|c|c|c|c|c|c|c|c|c|c|c|c|}
\hline \multirow[b]{3}{*}{ Scenario } & \multicolumn{5}{|l|}{ Piapung } & \multicolumn{5}{|l|}{ Missasso } & \multicolumn{5}{|l|}{ Dozanso } \\
\hline & \multirow[b]{2}{*}{$\begin{array}{l}\text { Time to } \\
\text { cross } \\
\text { threshold } \\
\text { (year) }\end{array}$} & \multicolumn{2}{|c|}{$\begin{array}{l}\text { Elimination } \\
\text { probability } \\
\text { (\%) }\end{array}$} & \multicolumn{2}{|c|}{$\begin{array}{l}\text { Recrudescence } \\
\text { probability (\%) }\end{array}$} & \multirow[b]{2}{*}{$\begin{array}{l}\text { Time to } \\
\text { cross } \\
\text { threshold } \\
\text { (year) }\end{array}$} & \multicolumn{2}{|c|}{$\begin{array}{l}\text { Elimination } \\
\text { probability } \\
(\%)\end{array}$} & \multicolumn{2}{|c|}{$\begin{array}{l}\text { Recrudescence } \\
\text { probability (\%) }\end{array}$} & \multirow[b]{2}{*}{$\begin{array}{l}\text { Time to } \\
\text { cross } \\
\text { threshold } \\
\text { (year) }\end{array}$} & \multicolumn{2}{|c|}{$\begin{array}{l}\text { Elimination } \\
\text { probability } \\
(\%)\end{array}$} & \multicolumn{2}{|c|}{$\begin{array}{l}\text { Recrudescence } \\
\text { probability (\%) }\end{array}$} \\
\hline & & $\begin{array}{l}\text { After } \\
5 \\
\text { years }\end{array}$ & $\begin{array}{l}\text { After } \\
35 \\
\text { years }\end{array}$ & $\begin{array}{l}\text { After } \\
5 \\
\text { years }\end{array}$ & $\begin{array}{l}\text { After } \\
35 \\
\text { years }\end{array}$ & & $\begin{array}{l}\text { After } \\
5 \\
\text { years }\end{array}$ & $\begin{array}{l}\text { After } \\
35 \\
\text { years }\end{array}$ & $\begin{array}{l}\text { After } \\
5 \\
\text { years }\end{array}$ & $\begin{array}{l}\text { After } \\
35 \\
\text { years }\end{array}$ & & $\begin{array}{l}\text { After } \\
5 \\
\text { years }\end{array}$ & $\begin{array}{l}\text { After } \\
35 \\
\text { years }\end{array}$ & $\begin{array}{l}\text { After } \\
5 \\
\text { years }\end{array}$ & $\begin{array}{l}\text { After } \\
35 \\
\text { years }\end{array}$ \\
\hline $\begin{array}{l}\text { Annual } \\
\text { MDA } \\
\text { upto 95\% } \\
\text { EP }\end{array}$ & 20 & 89 & 100 & 11 & 0 & 22 & 98 & 100 & 2 & 0 & 24 & 95 & 100 & 5 & 0 \\
\hline $\begin{array}{l}\text { Annual } \\
\text { MDA + } \\
\text { VC upto } \\
95 \% \text { EP }\end{array}$ & 14 & 97 & 99 & 3 & 1 & 19 & 99 & 99 & 1 & 1 & 20 & 98 & 99 & 2 & 1 \\
\hline $\begin{array}{l}\text { Annual } \\
\text { MDA } \\
\text { upto 95\% } \\
\text { EP, } \\
\text { endgame } \\
\text { VC }\end{array}$ & 20 & 89 & 100 & 11 & 0 & 22 & 98 & 100 & 2 & 0 & 24 & 95 & 100 & 5 & 0 \\
\hline $\begin{array}{l}\text { Annual } \\
\text { MDA } \\
\text { upto 95\% } \\
\text { EP, then } \\
\text { only VC } \\
\text { for next } \\
\text { 5yrs }\end{array}$ & 20 & 89 & 100 & 11 & 0 & 22 & 98 & 100 & 2 & 0 & 24 & 95 & 100 & 5 & 0 \\
\hline $\begin{array}{l}\text { Biannual } \\
\text { MDA } \\
\text { upto 95\% } \\
\text { EP }\end{array}$ & 11 & 22 & 99 & 78 & 1 & 12 & 63 & 99 & 37 & 1 & 13 & 45 & 100 & 55 & 0 \\
\hline $\begin{array}{l}\text { Biannual } \\
\text { MDA + } \\
\text { VC upto } \\
95 \% \text { EP }\end{array}$ & 8 & 85 & 98 & 15 & 2 & 11 & 96 & 99 & 4 & 1 & 11 & 94 & 98 & 6 & 2 \\
\hline $\begin{array}{l}\text { Biannual } \\
\text { MDA } \\
\text { upto 95\% } \\
\text { EP, } \\
\text { endgame } \\
\text { VC }\end{array}$ & 11 & 22 & 100 & 78 & 0 & 12 & 64 & 100 & 36 & 0 & 13 & 45 & 100 & 55 & 0 \\
\hline $\begin{array}{l}\text { Biannual } \\
\text { MDA } \\
\text { upto 95\% } \\
\text { EP, then } \\
\text { only VC } \\
\text { for next } \\
\text { 5yrs }\end{array}$ & 11 & 22 & 99 & 78 & 1 & 12 & 64 & 100 & 36 & 0 & 13 & 45 & 100 & 55 & 0 \\
\hline $\begin{array}{l}\text { Annual } \\
\text { IDA upto } \\
95 \% \text { EP }\end{array}$ & 13 & 29 & 99 & 71 & 1 & 16 & 75 & 100 & 25 & 0 & 17 & 63 & 100 & 37 & 0 \\
\hline $\begin{array}{l}\text { Annual } \\
\text { IDA + VC } \\
\text { upto 95\% } \\
\text { EP }\end{array}$ & 10 & 79 & 98 & 21 & 2 & 13 & 99 & 100 & 1 & 0 & 15 & 98 & 100 & 2 & 0 \\
\hline $\begin{array}{l}\text { Annual } \\
\text { IDA upto } \\
95 \% \text { EP, } \\
\text { endgame } \\
\text { VC }\end{array}$ & 13 & 29 & 100 & 71 & 0 & 16 & 75 & 100 & 25 & 0 & 17 & 63 & 100 & 37 & 0 \\
\hline $\begin{array}{l}\text { Annual } \\
\text { IDA upto } \\
95 \% \text { EP, } \\
\text { then only } \\
\text { VC for } \\
\text { next 5yrs }\end{array}$ & 13 & 29 & 99 & 71 & 1 & 16 & 75 & 100 & 25 & 0 & 17 & 63 & 100 & 37 & 0 \\
\hline
\end{tabular}




\section{Dynamics of LF recrudescence arising from applying the WHO and 95\% EP thresholds}

Figure 3 summarizes the recrudescence probabilities predicted in each of our six study sites by the end of the 5-year TAS period as a result of applying annual MDA alone for meeting either the WHO 1\% mf target (Fig. 3A) or the estimated site-specific 95\% EP mf thresholds (Fig. 3B, see also Table 2A, B). The panels also present the actual average levels of $\mathrm{mf}$ prevalence (red text over bars) and mf prevalence as a proportion of baseline prevalence (black text over bars) obtained in each site over this period once MDA was stopped after meeting either target. The results show that not only will the predicted recrudescence probabilities reach markedly higher levels when interventions are stopped once the $1 \% \mathrm{mf}$ target is achieved compared to when the $95 \%$ EP thresholds are crossed, but that the reinfection prevalence attained will also be significantly higher in the former scenario (mf prevalences reaching between 0.1 to $3 \%$ when the WHO target is used compared to just 0 to $0.01 \%$ in the case of using the $95 \%$ EP thresholds (Fig. 3).

Figure 4. Impact of annual MDA in DokanTofa, Nigeria (a) when annual MDA was continued to reach $1 \%$ mf threshold on 6 to 7 years old children, (b) when annual MDA was continued to reach $1 \% \mathrm{mf}$ threshold on 40 to 70 years old population, (c) when annual MDA was continued to reach $95 \%$ EP threshold on 6 to 7 years old children, (d) when annual MDA was continued to reach $95 \%$ EP threshold on $\mathbf{4 0}$ to $\mathbf{7 0}$ years old population. Gray lines indicate the model predictions whereas it changes color to red when it is going up and to green when it is going down towards elimination. Numbers in red and green color indicate the percentage of recrudescence probability and elimination probability of disease respectively. Blue solid, black dotted and solid horizontal lines are indicating $1 \% \mathrm{mf}$ threshold, $0.5 \% \mathrm{mf}$ threshold and model predicted $95 \%$ EP threshold respectively, whereas blue dotted and solid vertical lines indicating the time point to reach $1 \% \mathrm{mf}$ threshold and 5years of crossing the $1 \% \mathrm{mf}$ threshold (TAS period) respectively.

\section{Assessing validity of model forecasts using field data}

Field data to corroborate the above model predictions are still scare, but data from two studies allowed evaluation of the impact of adding vector control to MDA programmes as a measure for reducing infection recrudescence after stopping MDAs once prevalences are reduced to near $1 \% \mathrm{mf}$ prevalence ${ }^{22}$, and if this would indeed lead to sustained interruption of transmission once crossed and MDAs are stopped ${ }^{13,23-26}$. By contrast, surveillance data on the impact of annual MDA using DEC + ALB assembled from the commune of Leogane in Haiti allowed the direct investigation of whether using the WHO recommended 1\% $\mathrm{mf}$ threshold is sufficient to bring about LF transmission interruption and hence support the stopping of intervention ${ }^{13,23-26}$. In the latter setting, seven rounds of MDA were delivered starting from year 2000 with variable coverages and compliances and mf prevalence was reduced below the $1 \%$ threshold by the sixth year of treatments (in 2005); however, a subsequent survey carried out in whole population in year 2008 indicated that transmission was still ongoing in the community despite the apparent success of the applied MDAs ${ }^{13}$. Figure 5 depicts both the changes in mf prevalence in the community until year 7 post-MDA and the prevalences for both CFA (blue points/lines) and mf (red points/lines) at the post-MDA survey in year 2008. It also portrays the predictions of our model calibrated to the baseline $\mathrm{mf}$ data for the annual MDA interventions carried out in the commune (by taking also into account the actual coverages attained annually). These results provide important empirical evidence indicating that the WHO $1 \% \mathrm{mf}$ threshold has not led to interruption of LF transmission in this setting, and indeed that, as the model forecasts show (and further mimicking the theoretical results above), the use of this threshold will instead result in significant corresponding resurgences of infection if such arbitrarily thresholds are used for deciding the stopping of interventions.

In the study by Sunish et $\mathrm{al}^{22}$, on the other hand, one group of villages received two annual MDAs of DEC + IVM), while Group B villages received the same MDA schedule in combination with VC, chiefly using expanded polystyrene beads (EBP), biolarvicide and larvivorous fish (Table 3). MDAs were stopped after two annual treatments and the communities were resurveyed for infection levels three years after stoppage of MDAs in both groups of villages. VC, however, continued in the Group B villages following cessation of MDA. The results from the post-treatment surveys showed that VC preserved the effects of MDA while resurgences occurred in Group A villages (Table 4). Overall, continuing with vector control suppressed the mf prevalence attained three years following stoppage of MDA in the Group B villages by up to $55.8 \%$ compared to the mf prevalence reached in Group A (Table 3 ). Figure 6 shows the corresponding model predictions in comparison with observed data, and highlights that the theoretical findings described above regarding the impact of using supplementary vector control for both retaining the gains of MDA and arresting LF recrudescence may indeed operate in the field.

Table 3

Pre- and post-treatment data for two groups of villages at Tirukoilur in India.

\begin{tabular}{|c|c|c|c|}
\hline \multirow[t]{2}{*}{ Treatment Groups } & \multicolumn{3}{|l|}{ M\&E data 22} \\
\hline & $\begin{array}{l}\text { Pre-treatment (1994 October- } \\
\text { December) mf positive (\%) (no. } \\
\text { of sample) }\end{array}$ & $\begin{array}{l}\text { First survey (1997 October- } \\
\text { December) mf positive (\%) (no. } \\
\text { of sample) }\end{array}$ & $\begin{array}{l}\text { Second survey (1999 April- } \\
\text { June) mf positive (\%) (no. of } \\
\text { sample) }\end{array}$ \\
\hline $\begin{array}{l}\text { Group A (MDA alone for } 1995 \text { (June-August) and } \\
1996 \text { (July-September) }\end{array}$ & $15.19(724)$ & $1.81(609)$ & $4.74(591)$ \\
\hline $\begin{array}{l}\text { Group B (MDA+ VC for } 1995 \text { (June-August) and } \\
1996 \text { (July-September) and VC up to } 1998 \\
\text { (December)) }\end{array}$ & $15.09(795)$ & $1.24(645)$ & $2.08(673)$ \\
\hline
\end{tabular}

\section{Discussion}

Our major goal in this study was to undertake a model-based evaluation of the implications of using the current WHO recommended TAS criteria for determining if LF transmission interruption has been achieved permanently in treated communities. We also assessed and compared the effectiveness of various MDA intervention regimens with and without inclusion of vector control for ensuring the sustained achievement of parasite elimination while also suppressing the probability of infection recrudescence. The resolution of these questions pertaining to making reliable intervention stopping decisions are becoming urgent as many national LF programs begin to enter into the endgame or pre-elimination phase of interventions ${ }^{2,28}$, and need guidance as to when 
to cease implemented interventions or switch to enhanced strategies to ensure the sustained elimination of the disease. Our analysis indicates that ultimately the making of such stopping decisions and hence the effectiveness of an epidemiological assessment strategy for evaluating the achievement of LF transmission interruption will rely on the setting of targets the crossing of which will lead unequivocally to cessation of transmission ${ }^{9,10}$. While the WHO-set targets in the TAS protocol reflect both antecedents gained during the successful LF program implemented in China 29,30 and the goal of using a practical, operational, threshold that can be statistically measured, this study demonstrates that elimination thresholds for different observable indicators need to be set on the basis of the principles of transmission dynamics, particularly in relation to infection values denoting the approach of critical transitions in infectious disease systems 9,10,31, if the correct intervention stopping decisions are to be made. In addition, we highlight how the reliable design of surveillance protocols for assessing termination of parasite transmission successfully, including setting of extinction thresholds, requires the coupling of data-driven models describing the local processes of transmission to observable measures of infection in a given endemic setting. The simulations carried out in this work have also shed light on the relative values of current and proposed interventions for ensuring LF elimination and suppression of recrudescence in relation to the use of threshold targets designed for operational purposes versus those obtaining in a site on the basis of the underlying site-specific dynamic processes of parasite transmission and extinction.

We began the examination of the impact of the WHO-supported TAS thresholds by comparing the intervention outcomes that can be expected from using these thresholds to those arising from the use of the corresponding breakpoint mf prevalences estimated in each of our study sites over both the short-term TAS period of 5 years post stoppage of interventions and over a longer 20 years of intervention stoppage. The results arising from the application of the sitespecific LF models calibrated to baseline mf prevalence data in each of our study sites to predict the effects of the actually applied annual MDAs (using IVM + ALB or IVM + DEC) in these sites (based on recorded average coverages) were evaluated first in this regard (Table 4). These indicate, firstly, as we have shown previously ${ }^{9,10}$, that in contrast to the WHO recommended $1 \% \mathrm{mf}$ threshold to be applied commonly in all settings, the $95 \%$ EP mf threshold values predicted by the present locality-specific LF models are significantly lower than the currently-set TAS threshold. Importantly, the latter are also highly variable between sites casting further question on the recommendation of the use of a single globally-applicable threshold in the current TAS surveillance strategy.

The outcomes of these features of LF extinction dynamics in the design and use of threshold targets for assessing the probabilities of achieving both parasite elimination and recrudescence were then accessed initially over a short-term (after 5 years of MDA stoppage) from the carrying out of the MDA interventions implemented in each of the present study sites (see Table 1). Thus, while using the operationally convenient $1 \%$ mf threshold set by WHO would invariably lead to shorter durations of annual MDA required for its breaching in each site, the calculated probabilities of transmission interruption that would be achieved would be low or moderate ranging from as low as only $4-50 \%$ (Table $1 \mathrm{~A}$ ) with the subsequent probability of recrudescence ranging from $50 \%$ to as high as $96 \%$ even just after the 5-year TAS period. Note that probabilities of recrudescence were calculated in this study by enumerating the proportion of the 500 SIR models selected from fitting of the LF model to baseline mf prevalence data in each site demonstrating positive slopes after the cessation of applied interventions. However, as Fig. 3 illustrates, the range in prevalence values that may be expected following intervention stoppage after crossing the $1 \%$ mf threshold could also be wide and reach high levels (between 0.1 to $2.9 \%$ among the sites) just after 5 years post-treatment, suggesting that the present sites would most likely fail the current TAS criteria (sustained suppression of mf prevalence $<1 \%$ over the period of TAS survey bouts). By contrast, while it will take significantly and variably longer (up to 19 years of annual MDA depending on baseline $\mathrm{mf}$ prevalence and dynamically-occurring thresholds) to cross the sitespecific $95 \%$ EP mf thresholds. But once crossed, our results show that very high probabilities of parasite elimination (>98\%) and consequently very low probabilities of infection recrudescence (1 to 11\%) will be achieved over the 5 -year TAS period if these critical points arising from the learned LF system dynamics in a site are used as MDA elimination targets in the present sites (Table 1B). The data presented in Fig. $3 \mathrm{~b}$ further shows that the infection prevalences achieved immediately following the stoppage of interventions over the 5-year recommended TAS surveillance period will also be low and largely around or significantly below $1 \% \mathrm{mf}$ prevalence $(<0.01 \%)$ meaning that all the present sites will likely pass the TAS criteria for stopping interventions if the model-estimated $95 \% \mathrm{EP}$ mf threshold for each site is used as MDA elimination targets.

The second set of major simulations carried out in this study was focused on assessing the relative effectiveness of key currently applied and proposed MDAbased strategies with and without vector control for sustaining parasite elimination and suppression of infection recrudescence over the long-term when either of the WHO set $1 \% \mathrm{mf}$ threshold or the site-specific $95 \%$ EP thresholds estimated in each of the present study sites was used as the LF transmission interruption target. The predictions from this scenario-based simulations on the one hand support the major conclusions gleaned from the results in Table 1 , viz. that using the $1 \% \mathrm{mf}$ threshold would drastically reduce the number of years/cycles of annual interventions required to breach this target in each site (Table 2A) compared to crossing the corresponding 95\% EP mf thresholds (Table 2B). However, an intriguing result was the finding that while switching to biannual MDA and annual IDA with or without inclusion of vector control can significantly reduce the number of years of intervention required to cross the $1 \%$ $\mathrm{mf}$ threshold in comparison with the implementation of annual single MDA alone in each site, the probabilities of elimination achieved by meeting this threshold by the longer duration annual MDA was higher than that obtained from the application of relatively faster-acting biannual MDA or annual IDA alone strategies in these sites (Table 2A). The converse was true for the calculated recrudescence probabilities, with values reaching as high as $100 \%$ in the low prevalence sites when biannual MDA or IDA alone are used. This is an unexpected finding and hints at complex trade-offs between the faster ability of these more effective drug regimens (biannual MDA or annual IDA) to hasten the meeting of a relatively high valued threshold set primarily for operational reasons balanced by their inability to push infection further to elimination and thus reduce infection recrudescence. Interestingly, this result also suggests that annual MDA may have a greater impact in affecting higher elimination and lower recrudescence presumably as a result of its longer cummulative biological effects on the worm population if the $1 \% \mathrm{mf}$ threshold is used, highlighting the need for fully addressing the implications of using arbritary, untested, thresholds in making parasite intervention stopping decisions ${ }^{9,31}$.

By contrast to the differential outcomes found for the ability of the investigated MDA-based strategies to affect parasite elimination and suppress infection recrudescence when the $1 \% \mathrm{mf}$ prevalence threshold is used as an elimination target, the use of the site-specific $95 \%$ EP thresholds invariably led to high probabilities of infection elimination (close to $100 \%$ across the study settings) and near zero probability of infection recrudescence (close to 0 or $1 \%$ ) for the majority of the strategies investigated here. The results, however, showed that as for the case for using the $1 \%$ mf threshold as the elimination target

Page 9/17 
(Table 2A), switching from annual MDA to biannual MDA and IDA will generally lead to savings in the number of years of interventions required, although as expected compared to the years for crossing the $1 \%$ threshold the years of interventions required by all the investigated strategies to breach the significantly lower $95 \%$ EP $\mathrm{mf}$ threshold will be considerably longer (between 8 to 24 years of intervention depending on strategy and pre-intervention endemicity (Table 2B)).

The model predictions regarding the impact of including vector control into the MDA strategies we studied suggest that the outcomes may be complicated with the use of

this integrated strategy for accelerating and sustaining LF elimination, suggesting the need to be appreciative of the subtleties in the use of this approach. Thus, while combining VC with MDA from the start until the $1 \% \mathrm{mf}$ threshold is reached is shown not to improve neither the time to cross this threshold nor the elimination or recrudescence probabilities calculated compared to the use of MDA alone, irrespective of the MDA strategy modelled (Table 2A), while including VC after the achievement of this threshold by MDA can have a dramatic effect in increasing the probability of elimination to $71 \%-99 \%$ and reducing the corresponding probability of recrudescence (0-29\%) irrespective of site or MDA strategy for the longer term 20-year period (Table 2A). This is a major and unexpected finding and crucially implies that the WHO-set $1 \% \mathrm{mf}$ threshold for stopping MDAs can be used in by LF programs provided it is coupled with the inclusion of $\mathrm{VC}$ after this threshold is reached. This impact of long-term post-MDA inclusion of VC for ensuring parasite elimination and preventing infection recrudescence alongside the use of the $1 \% \mathrm{mf}$ threshold furthermore was robust irrespective of variations in local transmission conditions (Table $2 \mathrm{~A}$ ), implying as we have demonstrated previously ${ }^{10}$ that this strategy could also serve as a method for overcoming the observed between-site heterogeneity in the transmission of LF.

By direct contrast to the results for the above impact of post-MDA VC when used with the $1 \%$ mf threshold, the outcomes given in Table $2 B$ show that using MDA alone may be sufficient to bring about sustained parasite elimination if the $95 \%$ EP thresholds are used. These results indicate overall that national LF programs must now seriously consider integrating existing VC programs, such as the malaria bednets ${ }^{32,33}$, to supplement LF MDA programs if the currently used WHO infection elimination targets are continued to be used in LF elimination programs. Indeed, plans should be devised to incorporate such VC over the longer-term even after TAS is passed (perhaps up to 10 years at least post-TAS) if we are to ensure LF is sustainably eliminated if programs continue to use the present WHO targets. Using the 95\% EP targets as estimated here will obviate this need but it will take longer cycles of MDA to breach these thresholds (between 8-24 years depending on MDA regimen and inclusion of $\mathrm{VC}$ ), compared to using the $1 \% \mathrm{Mf}$ threshold (Tables $2 \mathrm{~A}, 2 \mathrm{~B}$ ). However, this needs to be balanced by the need for long-term VC (perhaps upto 10 years post MDA or 11 to 19 years of intervention overall) to ensure that transmission interruption is sustained once the latter threshold is crossed. However, note switching to biannual MDA or IDA will decrease the number of years drug treatments required to cross the $95 \% \mathrm{mf}$ threshold significantly to between 11 to 17 years (Table 3B) or similar to the years of MDA required when using MDA plus long-term VC in the case of applying the $1 \% \mathrm{Mf}$ threshold as described above. This means that logistical or feasibility challenges of applying longer-term MDA versus pursuance of long-term VC in different settings should then dictate the choice of the usable elimination target by LF national programs. Our modelling of the recrudescence dynamics of $\mathrm{mf}$ following the meeting of the $1 \%$ versus $95 \%$ EP threshold, however, indicates another important utility of using the latter threshold, viz. that while $\mathrm{mf}$ prevalence might meet the TAS criteria of being lower than the $0.5 \%$ prevalence target in the $6-7$ year-old TAS monitoring group during the 5-year TAS assessment period, it will both remain above and continue to increase above this threshold in the older population with time (Fig. 4A,B) signifying that transmission will continue in the community despite meeting the current TAS criteria. Using the $95 \%$ EP threshold, however, will ensure that predicted recrudescence rates will be insignificant and infection will decline to zero over time for both the child and older populations once these thresholds are breached and MDA is discontinued.

Our conclusions are clearly dependent on the capacity of our data-driven modelling approach to capture real-word observations reasonably well. We carried out two sets of validation exercises to ascertain the validity of our predictions. The first assessed the predictive ability of our data-fitted local models for both reproducing baseline LF infection and for predicting the impacts of the annual MDAs applied in each of the present study sites on infection prevalence. The results shown in Fig. 1 and Table 1 highlight that apart for two villages (Piapung and Kirare sites), the estimated locally applicable LF models are able to reproduce both infection prevalence at baseline and the corresponding observed changes in the data due to the applied MDA interventions in our study villages, heightening the confidence that our data-driven modelling approach may provide a sound basis for the results presented here on the likely outcomes of using the WHO TAS criteria as a means for quantifying site-specific interruption of LF transmission. This predictive ability of our modelling approach to mimic real-world LF transmission and control dynamics is further underscored by the modelling results shown in Figs. 5 and 6 and in Table 3 pertaining to field studies on the impact of supplementarty VC 22 and use of the WHO-propposed pre-TAS $1 \%$ mf threshold as a decision point for moving into the TAS phase ${ }^{13}$. Although carried out over relatively short intervention periods, the observations from these studies match model predictions indicating both that using a globally-fixed 1\% Mf prevalence may be sufficient to break LF transmission (Fig. 5) and that implementing VC into MDA programs can preserve the effects of MDA (Table 3, Fig. 6). These validations of the predictive performance of our data-driven modelling approach enhances the reliability of the results presented here, although analysis of longer-term data from more settings would further increase the validity of the conclusions regarding elimination thresholds, the TAS protocol, and interventions to ensure LF elimination made above.

In conclusion, our study has shown that using the right thresholds will be important for deciding if we are obtaining the right results, viz. in the present case whether applied LF interventions are able to sustainably interrupt community transmission of the parasite. We have also shown how various current and proposed MDA strategies could interact with the WHO TAS threshold and criteria for assessing transmission interruption versus the use of model-estimated elimination thresholds in this regard, and have identified optimal interventions for meeting either threshold. Specifically, we indicate that if the WHO-TAS threshold and criteria for evaluating transmission interruption is to be used, then overall it is imperative that long-term VC is applied post-stoppage of MDA to ensure that this interruption is sustained long-term. By contrast, if the 95\% EP thresholds are used, then we show that once these thresholds are breached by MDA interventions the resulting transmission interruption will be permanent without any need for inclusion of long-term VC. However, the present paper shows that the use of these more naturally-applicable thresholds will crucially require linking measurements, analysis and models to advance their practical use. 
Given the challenges of applying this approach everywhere effectively, we conclude finally that the present WHO TAS criteria for assessing LF elimination could be used for operational purposes but only if long-term VC is actively included into LF programs once the $1 \%$ mf threshold is breached by MDA alone.

\section{Methods}

\section{Data}

Data on longitudinal changes in LF mf infection prevalences, and on MDA interventions carried out in six representative low, medium and high transmission sites (DokanTofa, Nigeria; Piapung, Nigeria; Mossasso, Mali; Kirare, Tanzania; Peneng, Papua New Guinea (PNG); Dozanso, Mali), were assembled from the published literature ${ }^{18-21}$ for use in calibrating and running simulations of the EPIFIL model in this study. The six sites were selected on the basis of providing data on the level of baseline mf prevalence, where DokanTofa and Piapung represent low prevalence sites (mf prevalence 1\%-15\%), Mossasso and Kirare embody medium prevalence sites ( $\mathrm{mf}$ prevalence 16\%-35\%), and Peneng and Dozanso, high prevalence sites (mf prevalence $>35 \%$ ) respectively, while also giving details on the required inputs for identifying the local LF models applicable to each individual site. These data inputs comprise information on the annual biting rate (ABR) and dominant mosquito genus, as well as MDA intervention details, including the relevant drug regimen used, frequency, and population coverages of the applied MDAs (Table 4).

Table 4

Mf prevalence survey and MDA data for six LF endemic sites.

\begin{tabular}{|c|c|c|c|c|c|c|}
\hline Setting* & Site & $\begin{array}{l}\text { Mosquito } \\
\text { Genus }\end{array}$ & $A B R^{b}$ & $\begin{array}{l}\text { Baseline mf } \\
\text { prevalence }\end{array}$ & $\begin{array}{l}\text { Regimen } \\
(\text { Efficacyc) }\end{array}$ & $\begin{array}{l}\text { Average MDA coverage /Observed MDA } \\
\text { rounds }\end{array}$ \\
\hline \multirow[t]{2}{*}{ Low } & $\begin{array}{l}\text { DokanTofa, Nigeria } \\
18\end{array}$ & Anopheles & $\begin{array}{l}300- \\
5000\end{array}$ & $5.0 \%$ & $\begin{array}{l}\text { IVM + ALB } \\
(99 / 9)\end{array}$ & $76 \% / 7$ \\
\hline & Piapung, Nigeria ${ }^{18}$ & Anopheles & $\begin{array}{l}300- \\
5000\end{array}$ & $9.9 \%$ & $\begin{array}{l}\text { IVM + ALB } \\
(99 / 9)\end{array}$ & $77 \% / 7$ \\
\hline \multirow[t]{2}{*}{ Medium } & Mossasso, Mali ${ }^{19}$ & Anopheles & 605.9 & $20.2 \%$ & $\begin{array}{l}\text { IVM + ALB } \\
(99 / 9)\end{array}$ & $75 \% / 6$ \\
\hline & Kirare, Tanzania 20 & Anopheles $^{a}$ & 2090 & $26.1 \%$ & $\begin{array}{l}\text { IVM + ALB } \\
(99 / 9)\end{array}$ & $66 \% / 6$ \\
\hline \multirow[t]{2}{*}{ High } & Peneng, $\mathrm{PNG}^{21}$ & Anopheles & 8194 & $66.7 \%$ & $\begin{array}{l}\text { DEC + IVM } \\
(95 / 6)\end{array}$ & $69 \% / 5$ \\
\hline & Dozanso, Mali ${ }^{19}$ & Anopheles & 605.9 & $40.0 \%$ & $\begin{array}{l}\text { IVM + ALB } \\
(99 / 9)\end{array}$ & $75 \% / 6$ \\
\hline
\end{tabular}

*Low: mf prevalence 1\%-15\%, Medium: mf prevalence 16\%-35\%, High: mf prevalence > 35\%

${ }^{a}$ Transmission in Kirare is by both Anopheles and Culex mosquitoes, but models based on the dominant species (Anopheles) was used in this study. The ABR represents the combined biting rate.

${ }^{\mathrm{b}}$ In the model simulations, the allowed ABR range was informed by the observed ABRs reported here.

${ }^{\mathrm{C}}$ Drug efficacy figures denote the proportionate instantaneous $\mathrm{mf}$ kill rates and the duration of adult worm sterilization in months

The model

The EPIFIL LF transmission model used this study has been previously described in full elsewhere $9,10,16$. The model simulates LF transmission in a population by accounting for key biological and intervention processes, such as impacts of vector density, the life cycle of the parasite, age-dependent exposure, density-dependent mechanisms, infection aggregation, and the outcomes arising from drug treatments as well as vector control. In brief, the population dynamics of filarial infection are represented as coupled differential equations describing changes in five state variables pertaining to infection in both human and vector host populations. In the human host, the pre-patent and patent worm loads ( $P$ and W, respectively), mf (M) and CFA (A) intensity and prevalence, and a measure of acquired immunity $(I)$ to the parasite moderated by the total worm load $\left(P+W=W_{T}\right)$ are formulated as partial differential equations over time $(t)$ and host age (a). In the mosquito host, the burden of infective $L 3$ stage larvae $(L)$ is represented by a single state variable $\left(L^{*}\right)$ formulated as an ordinary differential equation integrated over time ( $\mathrm{t}$ ) given that this stage is assumed to reach equilibrium quickly owing to the faster time scale of infection dynamics in the vector compared to the human. The model is vector genus-specific ${ }^{9,31}$, but given that the dominant vector genus in all the present study settings was Anopheles (Table 4), the sub-model describing the uptake and development of mf into L3 larvae for these mosquitoes was applied in the present analysis. The structure, parameters and functions of this anopheline-based EPIFIL model are detailed fully in S1 Supplementary Information.

\section{Model calibration to site-specific infection data using Bayesian Melding}


We used a data-model assimilation technique based on the Bayesian Melding (BM) algorithm to calibrate and identify locality-specific LF transmission models based on the baseline mf prevalence data observed in each of our study sites ${ }^{9}$. This is done by "melding" the observed baseline mf data from each site with model-generated outputs in order to learn or parameterize models for describing the localized parasite transmission dynamics. The fitted models from each site were then used to quantify the various quantities of interest to this study, viz. estimations of mf breakpoints and threshold biting rates, predictions of the impact of various MDA interventions with and without vector control on mf prevalence, and calculations of the probabilities of transmission interruption and recrudescence from using the WHO-set TAS thresholds versus model-derived breakpoint values once mf prevalences are forecast to cross below these thresholds in each study site.

The BM procedure begins by first specifying a range of plausible parameter values to generate distributions of parameter priors. We then randomly sample from those prior distributions to generate 200,000 parameter vectors, which are then used with the observed ABR in a site to generate predictions of baseline age-specific pevalences. The Sampling Importance Resampling (SIR) algorithm is then used to select $N$ (typically $N=500$ ) parameter vectors, $\theta$, or models applicable to a site based on their likelihoods for describing the observed local baseline prevalence data. This BM fitting procedure normally relies on observed baseline age profiles of $\mathrm{mf}$ prevalence ${ }^{9}$, but, in the present analysis, these data were available only for DoakanTofa and Piapung, while overall community level $\mathrm{mf}$ prevalences were available for the other sites (Mossasso, Kirare, Peneng, Dozanso). In this scenario, the observed overall prevalences from these sites were transformed into theoretical age infection profiles using: 1) the national demographic profile applicable to the site in question, and 2) by conversion of the community-level $\mathrm{mf}$ prevalence to reflect either a plateau, concave or linear age-infection profile known typically to occur in LF endemic regions ${ }^{15}$. The derived age-prevalence infection data were then used in the model fitting procedures described above, which also effectively allowed the integration of partially observed data into the present LF model.

\section{Numerical stability analysis for quantifying infection breakpoints and vector biting thresholds}

A numerical stability analysis procedure, based on varying initial values of endemic infective larval density $\left(L^{*}\right)$ of each of the SIR selected model parameter sets or vectors, was used to calculate the distribution of $\mathrm{mf}$ prevalence breakpoints and the corresponding threshold biting rates (TBR) expected in each of the study communities $9,10,16,31,34$. Briefly, in this method, we begin by progressively decreasing the vector to human density ratio, $\mathrm{V} / \mathrm{H}$, from its original value to a threshold value below which the model always converges to zero $\mathrm{mf}$ prevalence, regardless of the values of the endemic infective larval density $L^{*}$. The product of the number of bites per mosquito, $\lambda$, and the newly found $\mathrm{V} / \mathrm{H}$ value is termed as the threshold biting rate (TBR). Once the threshold biting rate is discovered, the model at TBR will settle to either a zero (trivial attractor) or non-zero mf prevalence depending on the starting value of $L^{*}$. Thus, in the next step, while keeping all the model parameters unchanged, including the new $\mathrm{V} / \mathrm{H}$ and by starting with a very low value of $\mathrm{L}^{\star}$ and progressively increasing it in very small step-sizes we estimate the minimum $L^{\star *}$ below which the model predicts zero $\mathrm{mf}$ prevalence and above which the system progresses to a positive endemic infection state. Here, $L^{* *}$ thus represents the $L 3$ breakpoint density in the vector population ${ }^{9}$, whereas the corresponding $m f$ prevalence at the $L^{\star \star}$ value denotes the worm $/ \mathrm{mf}$ breakpoint ${ }^{31,35}$. Note that this procedure can also be used to estimate the mf prevalence breakpoints at the undisturbed $A B R$ values in a site. In this case, the estimated $\mathrm{mf}$ breakpoint prevalences will always be lower than the corresponding maximal values at TBR ${ }^{9,10}$. The collections of $\mathrm{mf}$ breakpoint prevalences from the SIR selected parameters in a site are then used to derive the infection extinction thresholds signifying various probabilities of elimination. Note that in this study, we focused on the $95 \%$ elimination probability (EP) threshold value to serve as model-derived elimination targets for carrying out the intervention simulations described below.

\section{Calculating extinction and recrudescence probabilities}

We calculated the probability that LF extinction has been achieved in each study site due to the applied intervention by quantifying the proportion of the bestfit SIR model prevalences that were declining or declined to zero (ie. models giving rise to $\mathrm{mf}$ prevalence curves with negative slopes) by the end of the 5 or 20 year simulation period following crossing of either the pre-TAS threshold of $1 \% \mathrm{mf}$ or the predicted site-specific $95 \%$ EP threshold values, respectively. The recrudescence probability for a given study site was similarly calculated as the proportion of the total SIR selected model runs that managed to revive and generate positive increases in $\mathrm{mf}$ prevalence (ie. give rise to $\mathrm{mf}$ curves with positive slopes) by the end of each of the above simulation periods once MDAs are stopped.

\section{Modelling the impact of MDA and vector control interventions.}

Interventions were modelled by using the SIR-selected parameter vectors/models for simulating the impacts of both currently used as well as proposed MDAbased intervention strategies in reducing the observed baseline LF prevalence in each site to below either the global TAS (1\% mf prevalence) or site-specific 95\% EP thresholds. When simulating these interventions, the observed MDA regimens and coverages followed in each site were used (Table 1), while MDA was assumed to target all residents aged 5 years and above. While the drug-induced $\mathrm{mf}$ kill rate and the duration of adult worm sterilization were fixed among the models (Table 4), the worm kill rate was left as a free parameter to be estimated from the post-intervention data to account for uncertainty in this drug efficacy parameter. For making $\mathrm{mf}$ prevalence forecasts beyond the observations made in each site, predictions arising from the impacts of MDA simulated with and without vector control were carried out for 5 years and 20 years after the stoppage of MDA in each site at the MDA coverages listed in Table 4. Three different MDA regimens: (i) annual MDA with ivermectin and albendazole (IVM + ALB) or diethylcarbamazine and ivermectin (IVM + DEC) as applied in each site (Table 4), (ii) biannual MDA with the above regimens, and (iii) annual triple drug MDA (IDA: combined ivermectin, diethylcarbamazine and albendazole) were modelled with and without vector control in this study to provide a comparison of the effectiveness of these drug regimens for affecting LF elimination. In these simulations, MDAs are stopped after achieving either the $1 \% \mathrm{mf}$ TAS threshold or the model predicted $95 \%$ EP threshold in each modelled site but simulations of subsequent changes in mf prevalence with or without vector control (VC) for the next 20 years were continued to evaluate the probability of LF elimination and the risk of recrudescence of the infection respectively over both the shorter 5 -year TAS period and over the longer-term 20 years period (using the assessment methods for calculating the occurrence of either of these events described above). VC is modelled in terms of the impact of long-lasting 
insecticidal nets (LLINs) with $65 \%$ coverage following the equation given in Singh \& Michael ${ }^{9}$. The specific details concerning the different MDA-based scenarios investigated are as listed in Tables 2A and 2B.

Note that the estimated 95\% EP mf prevalence threshold at ABR was used when carrying out simulations of durations or timelines to break transmission by MDA alone strategies while the corresponding and comparatively higher 95\% EP thresholds (obtaining at TBR) for this indicator was employed when modelling the impact of including VC into MDA programs ${ }^{9}$. The MDA plus MDA and vector control model formulations, parameters and functions used to carry out these simulations are as described previously and provided in S1 Supplementary Information.

\section{Declarations \\ Data Availability}

All the data used in this work are provided in the main text in different tables with proper citation.

\section{Code Availability}

All the MATLAB codes used in this work are available in GitHub:

https://github.com/EdwinMichaelLab/LF-TAS-Project.git

\section{Acknowledgements}

This work was made possible by an internal grant from the University of South Florida. The funder had no role in study design, data collection and analysis, decision to publish, or preparation of the manuscript. The authors gratefully acknowledge the support of University of Notre Dame's Center for Research Computation for carrying out a part of the model runs using the MATLAB Parallel Computing Toolbox available on Computer Clusters.

\section{Author contributions}

E.M., S.S., and M.E.S. designed the study. S.S., E.M., and M.E.S. analyzed data. E.M. supervised the work. E.M., S.S. wrote the paper. E.M., S.S., and M.E.S. reviewed the draft.

\section{Competing interests}

The authors declare that they have no competing interests.

\section{References}

1. Fact Sheet: Lymphatic Filariasis [Internet]. Geneva, Switzerland: World Health Organization, Available: http://www.who.int/mediacentre/factsheets/fs102/en/ (2012).

2. Rebollo, M. P. \& Bockarie, M. J. Toward the elimination of lymphatic filariasis by 2020: treatment update and impact assessment for the endgame. Expert Rev. Anti-infect. Ther. 11, 723-731 (2013).

3. Irvine, M. A. et al. Effectiveness of a triple-drug regimen for global elimination of lymphatic filariasis: a modelling study. Lancet Infect. Dis. 17, 451-458 (2017).

4. World Health Organization, Global Programme to Eliminate Lymphatic Filariasis: Monitoring and epidemiological assessment of mass drug administration: a manual for national elimination programs. Geneva. 22-33, (2011).

5. Lau, C. L. et al. Detecting and confirming residual hotspots of lymphatic filariasis transmission in American Samoa 8 years after stopping mass drug administration. PLoS Negl. Trop. Dis. 11, e0005914 (2017).

6. Rao, R. U. et al. A comprehensive assessment of lymphatic filariasis in Sri Lanka six years after cessation of mass drug administration. PLoS Negl. Trop. Dis. 8, e3281 (2014).

7. Sheel, M. et al. Identifying residual transmission of lymphatic filariasis after mass drug administration: Comparing school-based versus communitybased surveillance-American Samoa, 2016. PLoS Negl. Trop. Dis. 12, e0006583 (2018).

8. Garchitorena, A. et al. Towards elimination of lymphatic filariasis in southeastern Madagascar: Successes and challenges for interrupting transmission. PLoS Negl. Trop. Dis. 12, e0006780 (2018).

9. Singh, B. K. \& Michael, E. Bayesian calibration of simulation models for supporting management of the elimination of the macroparasitic disease, lymphatic filariasis. Parasites Vectors 8, 1-26 (2015).

10. Michael, E. \& Singh, B. K. Heterogeneous dynamics, robustness/fragility trade-offs, and the eradication of the macroparasitic disease, lymphatic filariasis. BMC Med. 14, 1-23 (2016). 
11. Gambhir, M., Singh, B. K. \& Michael, E. The Allee effect and elimination of neglected tropical diseases: a mathematical modelling study. Adv. Parasitol. 87, 1-31 (2015).

12. Prada, J. M. et al. Elimination or resurgence: modelling lymphatic filariasis after reaching the $1 \%$ microfilaremia prevalence threshold. J. Infect. Dis. 221, S503-S509 (2020).

13. Boyd, A. et al. A community-based study of factors associated with continuing transmission of lymphatic filariasis in Leogane, Haiti. PLoS Negl. Trop. Dis. 4, e640 (2010).

14. Weiss, P. S., Michael, E. \& Richards Jr, F. O. Simulating a Transmission Assessment Survey: An evaluation of current methods used in determining the elimination of the neglected tropical disease, Lymphatic Filariasis. Int. J. Infect. Dis. 102, 422-428 (2021).

15. Smith, M. E., Singh, B. K. \& Michael, E. Assessing endgame strategies for the elimination of lymphatic filariasis: A model-based evaluation of the impact of DEC-medicated salt. Sci. Rep. 7, 1-12 (2017).

16. Michael, E. et al. Continental-scale, data-driven predictive assessment of eliminating the vector-borne disease, lymphatic filariasis, in sub-Saharan Africa by 2020. BMC Med. 15, 1-23 (2017).

17. Michael, E., Malecela-Lazaro, M. N., Kabali, C., Snow, L. C. \& Kazura, J. W. Mathematical models and lymphatic filariasis control: endpoints and optimal interventions. Trends Parasitol. 22, 226-233 (2006).

18. Richards, F. O. et al. Epidemiological and entomological evaluations after six years or more of mass drug administration for lymphatic filariasis elimination in Nigeria. PLoS Negl. Trop. Dis. 5, e1346 (2011).

19. Simonsen, P. E. et al. Lymphatic filariasis control in Tanzania: effect of repeated mass drug administration with ivermectin and albendazole on infection and transmission. PLoS Negl. Trop. Dis. 4, e696 (2010).

20. Simonsen, P. E. et al. Lymphatic filariasis control in Tanzania: effect of six rounds of mass drug administration with ivermectin and albendazole on infection and transmission. BMC Infect. Dis. 13, 335 (2013).

21. Singh, B. K. et al. Sequential modelling of the effects of mass drug treatments on anopheline-mediated lymphatic filariasis infection in Papua New Guinea. PLoS One 8, e67004 (2013).

22. Sunish, I. et al. Resurgence in filarial transmission after withdrawal of mass drug administration and the relationship between antigenaemia and microfilaraemia-a longitudinal study. Trop. Med. Int. Health 7, 59-69 (2002).

23. de Rochars, M. B. et al. The Leogane, Haiti demonstration project: decreased microfilaremia and program costs after three years of mass drug administration. Am. J. Trop. Med. Hyg. 73, 888-894 (2005).

24. Grady, C. A. et al. Endpoints for lymphatic filariasis programs. Emerg. Infect. Dis. 13, 608 (2007).

25. Mathieu, E. et al. Comparison of methods for estimating drug coverage for filariasis elimination, Leogane Commune, Haiti. Trans. R. Soc. Trop. Med. Hyg. 97, 501-505 (2003).

26. Mathieu, E. et al. Participation in three consecutive mass drug administrations in Leogane, Haiti. Trop. Med. Int. Health 11, 862-868 (2006).

27. Smith, M. E. et al. Predicting lymphatic filariasis transmission and elimination dynamics using a multi-model ensemble framework. Epidemics 18, 16-28 (2017).

28. Rebollo, M. P. \& Bockarie, M. J. Can lymphatic filariasis be eliminated by 2020? Trends Parasitol. 33, 83-92 (2017).

29. Sun, D.-J., Fang, Y., Huang, Y. \& Zhang, Y. Contributions to the lymphatic filariasis elimination programme and post-elimination surveillance in China by NIPD-CTDR. Adv. Parasitol. 110, 145-183 (2020).

30. Fang, Y. \& Zhang, Y. Lessons from lymphatic filariasis elimination and the challenges of post-elimination surveillance in China. Infect. Dis. Poverty $8,1-10$ (2019).

31. Gambhir, M. et al. Geographic and ecologic heterogeneity in elimination thresholds for the major vector-borne helminthic disease, lymphatic filariasis. BMC Biol. 8, 1-13 (2010).

32. Davis, E., Prada, J., Reimer, L. \& Hollingsworth, T. Modelling the Impact of Vector Control on Lymphatic Filariasis Programs: Current Approaches and Limitations. Clin. Infect. Dis. 72, S152-S157 (2021).

33. Bockarie, M. J., Pedersen, E. M., White, G. B. \& Michael, E. Role of vector control in the global program to eliminate lymphatic filariasis. Annu. Rev. Entomol. 54, 469-487 (2009).

34. Reimer, L. J. et al. Insecticidal bed nets and filariasis transmission in Papua New Guinea. N. Engl. J. Med. 369, 745-753 (2013).

35. Gambhir, M. \& Michael, E. Complex ecological dynamics and eradicability of the vector borne macroparasitic disease, lymphatic filariasis. PLoS One 3, e2874 (2008).

\section{Figures}



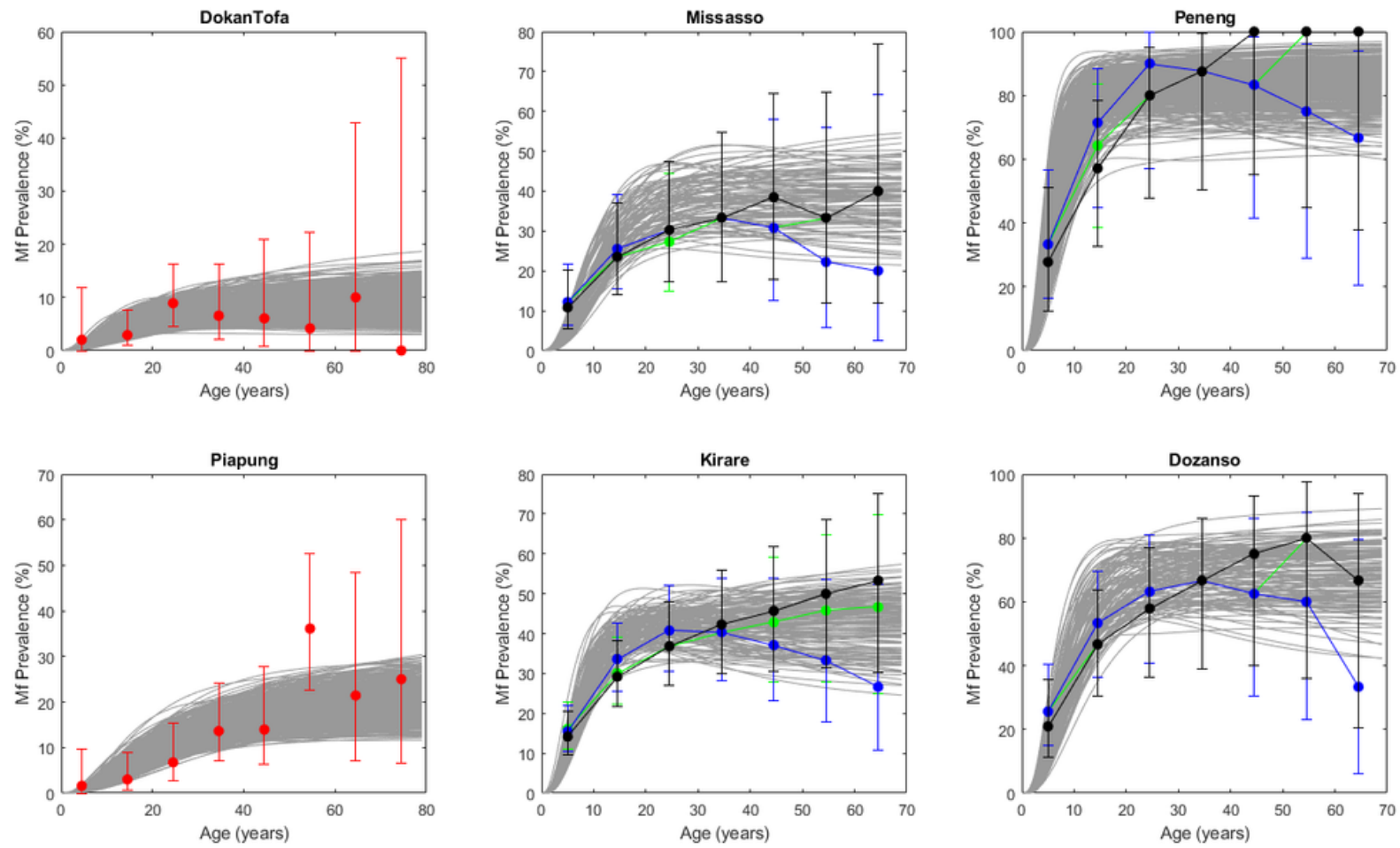

\section{Figure 1}

Validating models with baseline data for two low prevalence sites (DokanTofa and Piapung), two medium prevalence sites (Missasso and Kirare), and two high prevalence sites (Peneng and Dozanso). Red circles indicate the observed data and gray lines indicate the model predictions whereas black, blue and green circles joined by lines indicate constructed age prevalences. The age-stratified and overall Monte Carlo $\mathrm{p}$-values for the fits of baseline infection data for six study sites were evaluated (provided in Table 2 in S1 Supplementary Information).
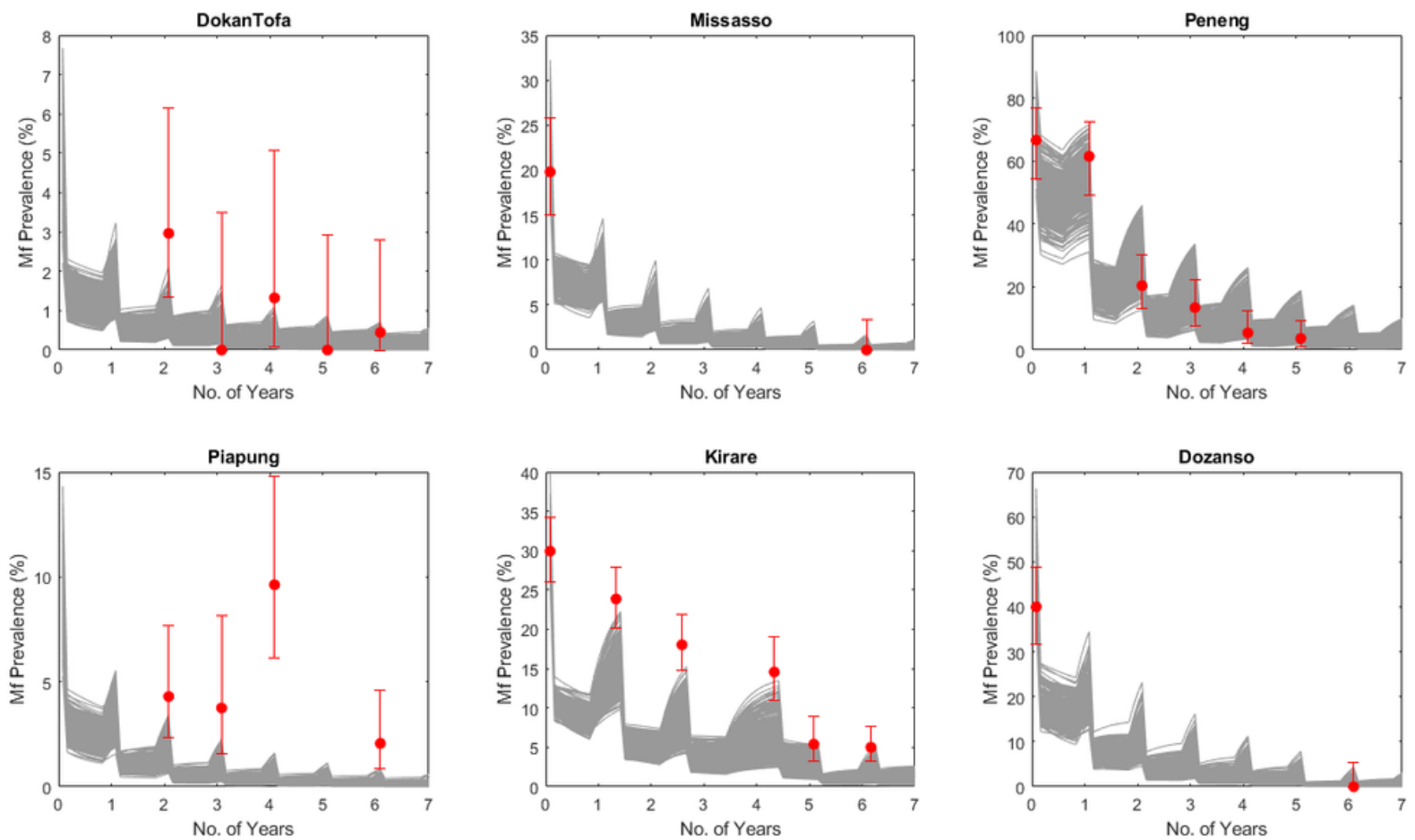

Figure 2 
Validating models with Intervention data for two low prevalence sites (DokanTofa and Piapung), two medium prevalence sites (Missasso and Kirare), and two high prevalence sites (Peneng and Dozanso). Red circles indicate the observed data and gray lines indicate the model predictions. The overall Monte Carlo $\mathrm{p}$ values for the fits of post MDA data for six study sites were evaluated (provided in Table 2 in S1 Supplementary Information).

(a)

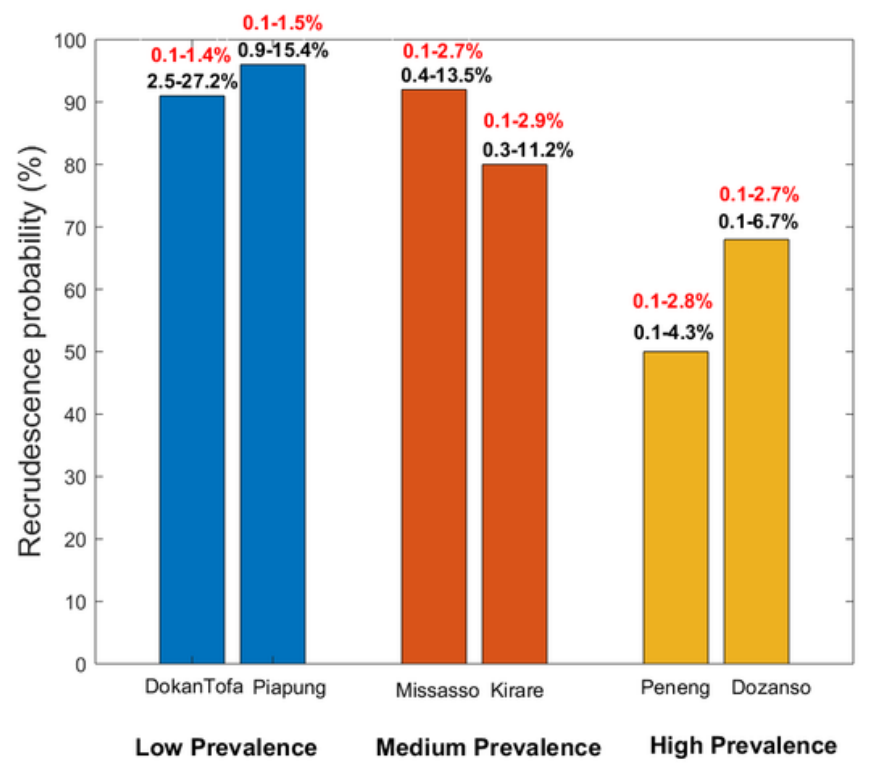

(b)

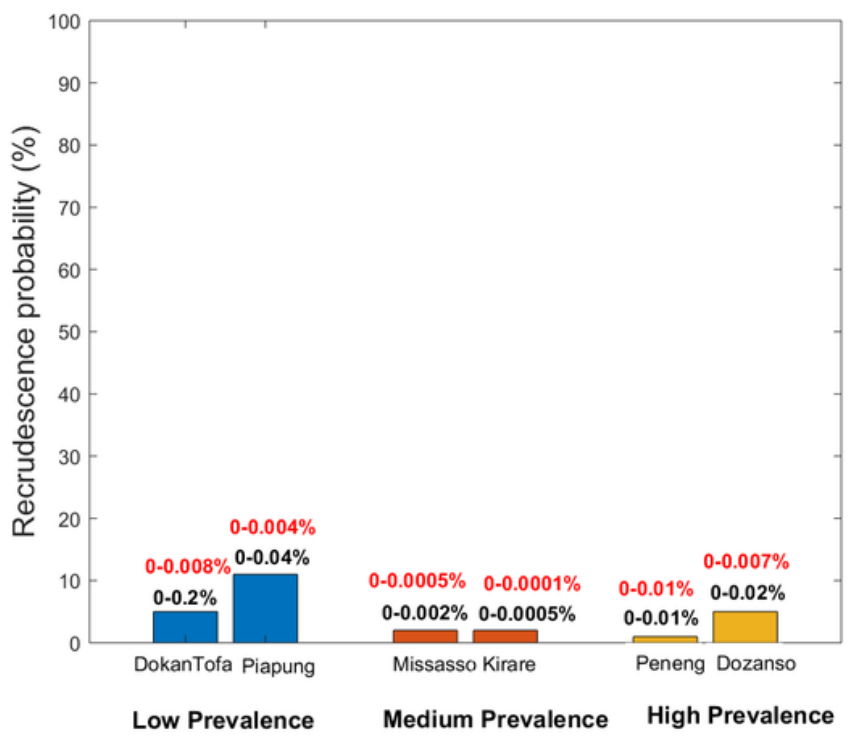

\section{Figure 3}

Bar diagram of recrudescence probability for two low prevalence (DokanTofa, Piapung), two medium prevalence (Missasso, Kirare) and two high prevalence (Peneng, Dozanso) study sites (a) after 5years of stopping annual MDA after crossing $1 \% \mathrm{mf}$ threshold, and (b) after 5years of stopping annual MDA after crossing $95 \%$ EP threshold. Figures in red above each bar indicate actual average levels of mf prevalence and the figures in black above each bar indicate the $\mathrm{mf}$ prevalence as proportion of baseline mf prevalence.

(a)

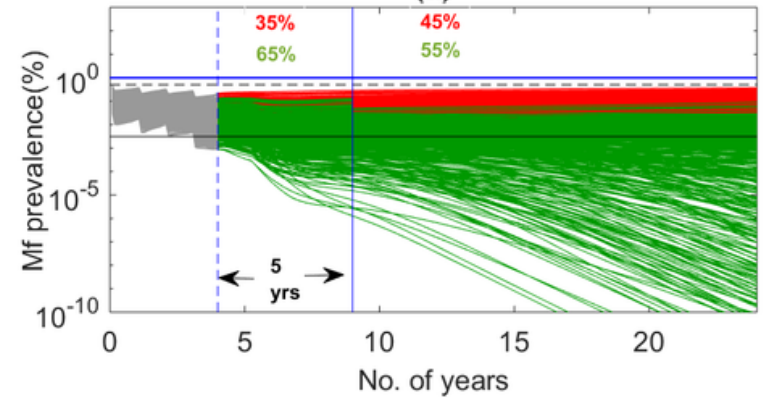

(c)

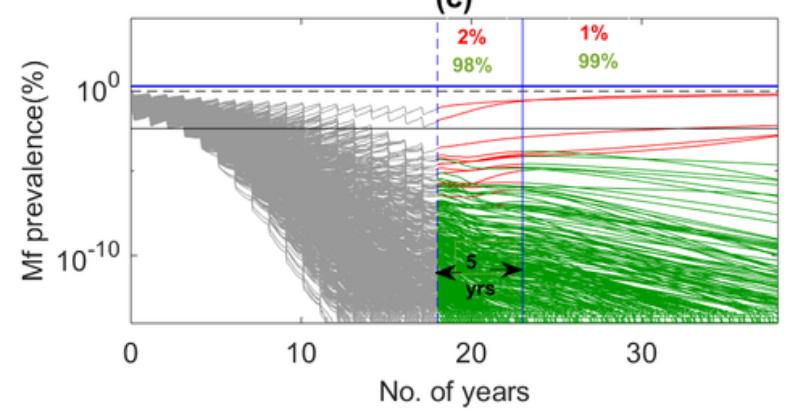

(b)

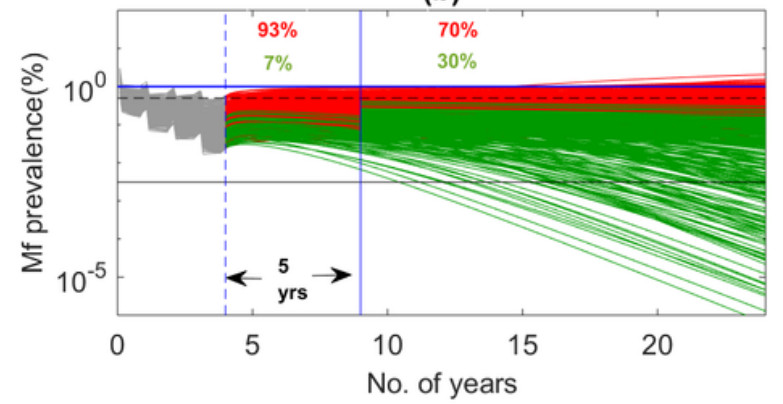

(d)

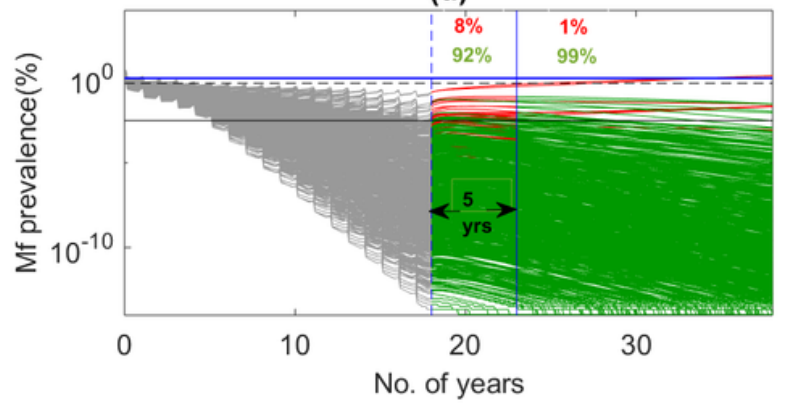

\section{Figure 4}

Impact of annual MDA in DokanTofa, Nigeria (a) when annual MDA was continued to reach $1 \%$ mf threshold on 6 to 7 years old children, (b) when annual MDA was continued to reach $1 \% \mathrm{mf}$ threshold on 40 to 70 years old population, (c) when annual MDA was continued to reach $95 \%$ EP threshold on 6 to 7 years old children, (d) when annual MDA was continued to reach $95 \%$ EP threshold on 40 to 70 years old population. Gray lines indicate the model predictions 
whereas it changes color to red when it is going up and to green when it is going down towards elimination. Numbers in red and green color indicate the percentage of recrudescence probability and elimination probability of disease respectively. Blue solid, black dotted and solid horizontal lines are indicating $1 \% \mathrm{mf}$ threshold, $0.5 \% \mathrm{mf}$ threshold and model predicted $95 \%$ EP threshold respectively, whereas blue dotted and solid vertical lines indicating the time point to reach $1 \% \mathrm{mf}$ threshold and 5 years of crossing the $1 \% \mathrm{mf}$ threshold (TAS period) respectively.

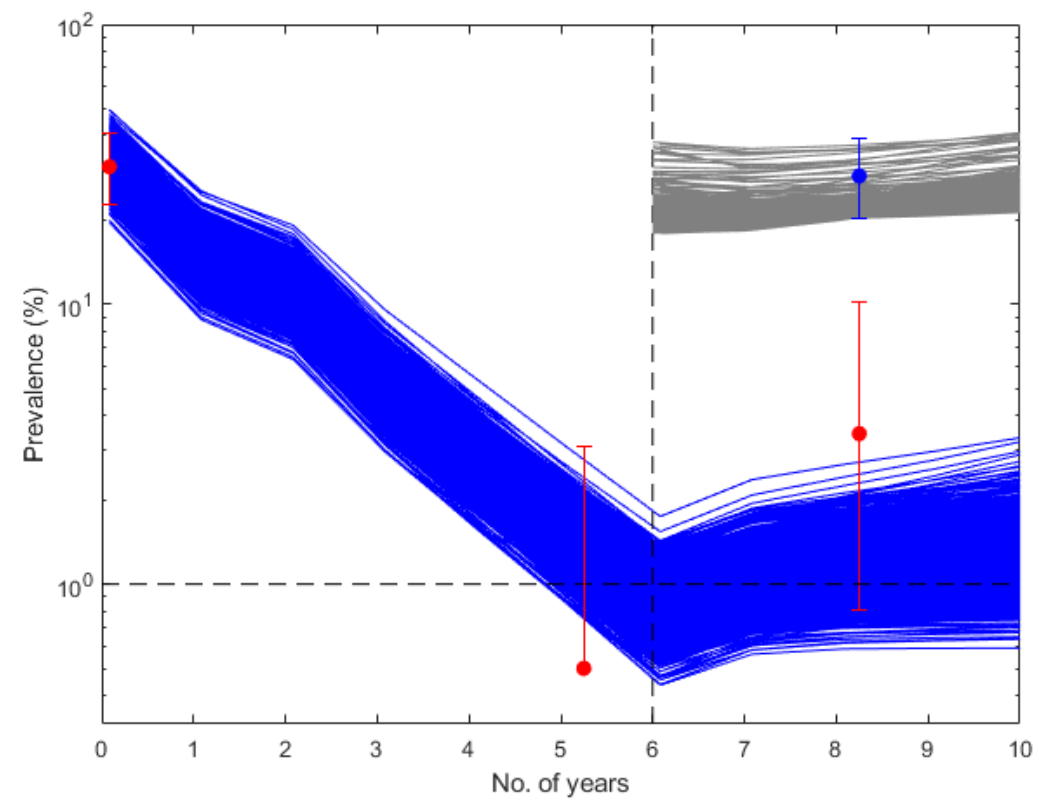

\section{Figure 5}

Model-predicted impact of MDA on mf prevalence during seven rounds of MDA and after stopping MDA and CFA prevalence at the post-MDA survey in year 2008 for whole community. Blue lines indicate the predicted impact of the interventions on mf prevalence whereas grey lines indicate the predicted impact of the interventions on CFA prevalence. Red dots indicate field data points for $\mathrm{mf}$ and blue dot indicates field data points for CFA. Horizontal and vertical black dotted lines indicate the WHO recommended $1 \% \mathrm{mf}$ threshold and the time point of stopping MDA respectively.
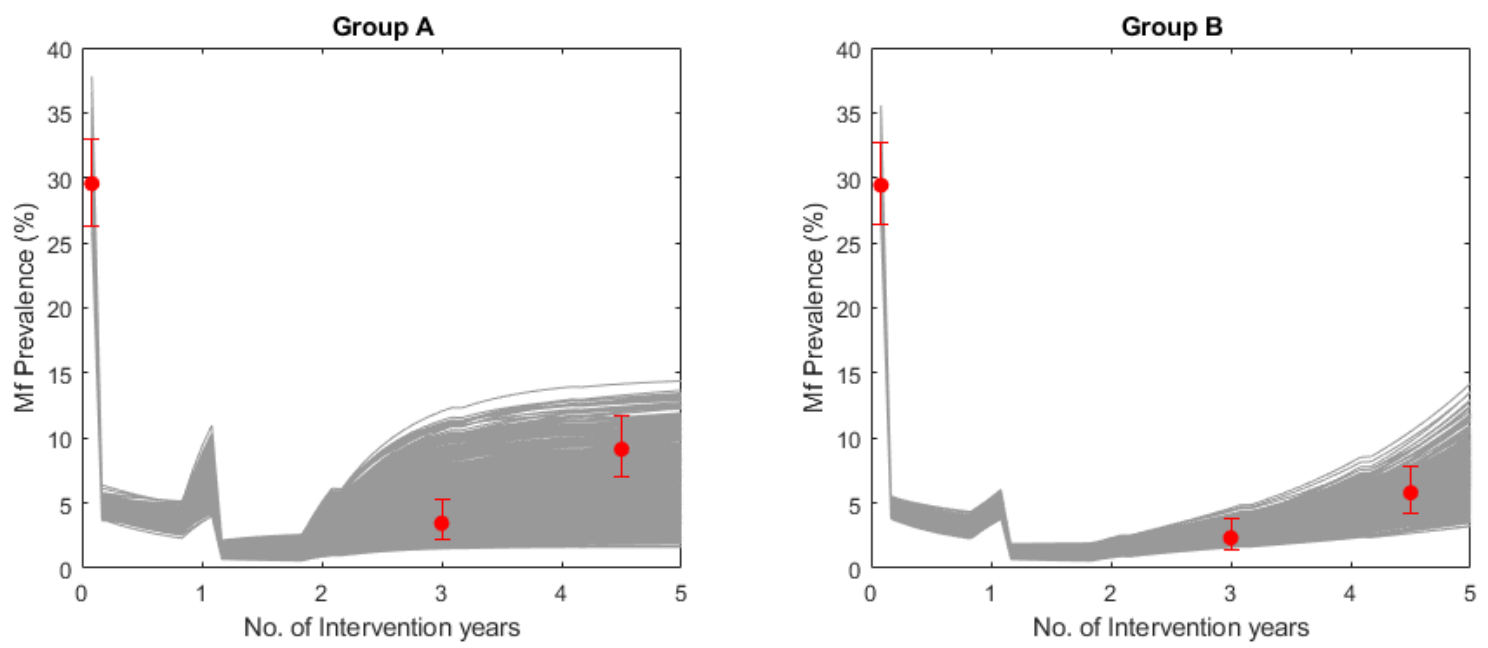

\section{Figure 6}

Model-predicted impact of MDA alone (Group A) and MDA with VC with continuation of VC (Group B) on mf prevalence. Grey lines indicate the predicted impact of the interventions on $\mathrm{mf}$ prevalence and red dots indicate field data points. To implement simulations of the impact of integrated vector management (IVM) interventions carried out in Group B we fitted a segmented exponential function for monthly biting rate (MBR): MBR = MBR0exp[a1t], with a $1<0$ for all $t$ when $V C$ is $O N$, otherwise $a 1>027$. Here we took $a 1=-5$ for our model simulation.

\section{Supplementary Files}

This is a list of supplementary files associated with this preprint. Click to download.

- S1SupplementaryinformationCB.docx 